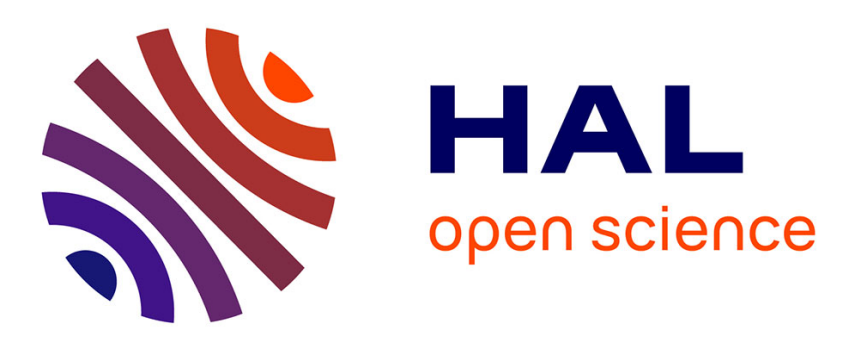

\title{
Smectic E side-chain liquid crystalline poly(methacrylates)
}

Robert Duran, D. Guillon, Ph. Gramain, A. Skoulios

\section{To cite this version:}

Robert Duran, D. Guillon, Ph. Gramain, A. Skoulios. Smectic E side-chain liquid crystalline poly(methacrylates). Journal de Physique, 1988, 49 (8), pp.1455-1466. 10.1051/jphys:019880049080145500 . jpa-00210826

\section{HAL Id: jpa-00210826 https://hal.science/jpa-00210826}

Submitted on 1 Jan 1988

HAL is a multi-disciplinary open access archive for the deposit and dissemination of scientific research documents, whether they are published or not. The documents may come from teaching and research institutions in France or abroad, or from public or private research centers.
L'archive ouverte pluridisciplinaire HAL, est destinée au dépôt et à la diffusion de documents scientifiques de niveau recherche, publiés ou non, émanant des établissements d'enseignement et de recherche français ou étrangers, des laboratoires publics ou privés. 
Classification

Physics Abstracts

$61.30-61.40-61.10$

\title{
Smectic E side-chain liquid crystalline poly(methacrylates)
}

\author{
R. Duran, D. Guillon, Ph. Gramain and A. Skoulios \\ Groupe des Matériaux Organiques, Institut Charles Sadron (CRM-EAHP), CNRS-ULP, 6 rue Boussingault, \\ 67083, Strasbourg Cedex, France \\ Institut de Physique et Chimie des Matériaux de Strasbourg, UM 380046 CNRS-ULP-EHICS, France
}

(Reçu le 29 janvier 1988, révisé le 11 avril 1988, accepté le 13 avril 1988)

\begin{abstract}
Résumé. - Une série homologue de trois poly(méthacrylates) liquides cristallins à groupes mésogènes latéraux a été étudiée par diffraction des rayons $\mathrm{X}$. Les groupes mésogènes constitués par des groupes $4^{\prime}-$ méthoxybiphénylyle étaient reliés au squelette macromoléculaire par des espaceurs souples de nature oligooxyéthyléniques. La structure smectique $\mathrm{E}$ monocouche de ces polymères a été analysée en termes de conformations du squelette macromoléculaire. Les groupes latéraux sont tous situés du même côté par rapport à la chaîne principale, et conduisent à des éléments de structure avant la forme de rubans. Les rubans sont assemblés côte à côte avec les groupes mésogènes pointant alternativement vers le haut et vers le bas.
\end{abstract}

\begin{abstract}
A homologous series of three side-chain liquid crystalline poly(methacrylates) with 4'methoxybiphenylyl pendant mesogenic groups and oligo(ethylene-oxide) flexible spacers has been studied by $\mathrm{X}$-ray diffraction. These polymers were shown to form a single layered smectic $\mathrm{E}$ structure, which has been analysed in terms of polymer chain conformation. The pendant groups were found to all hang on the same side of the polymer backbone to form ribbons, which are assembled side by side with an alternating up and down orientation of the pendant groups.
\end{abstract}

\section{Introduction.}

Side-chain liquid crystalline polymers have attracted considerable attention during the last few years [15]. Many articles have been published so far dealing with the synthesis of novel combinations of mesogenic groups, polymer backbones, flexible spacers, and aliphatic endgroups. Also, many articles have been devoted to the study of the general liquid crystalline behaviour of these polymers, by techniques such as optical microscopy and differential scanning calorimetry. As for structural determinations by $\mathrm{X}$-ray diffraction, a fair number of studies have been reported in the literature [6-9] ; but little systematic work has been carried out on homologous series of carefully characterized polymers. To contribute to this active field of research, our laboratory has recently undertaken a systematic study of side-chain liquid crystalline poly(methacrylates). The synthesis [10], the physico-chemical characterization [11], the thermal analysis [12], and some preliminary structural investigations [13-15] of these polymers have already been described and discussed.
The present paper is concerned with the detailed structural analysis of a short homologous series of poly(methacrylate) side-chain liquid crystalline polymers. After a thorough discussion of some structural concepts currently used in the field of side-chain polymers, several general considerations will be developed concerning the tacticity of polymer chains. In a second part, the samples and experimental Xray techniques used will be presented. In a third part, the X-ray results obtained will be described and analysed in terms of crystallographic structures and polymer chain conformations.

\section{Situation of the problem.}

Before discussing this work, it is worth recalling two main results which have been published to date in the literature for the structure of side-chain liquid crystalline polymers. The first is summarized by the ideas developed in a general paper by Finkelmann [3] and deals with the conformation of the polymer chain and the role of the flexible spacer. The second concerns the configuration of the pendant groups 
with respect to the polymer backbone in smectic mesophases and is presented in the papers by Shibaev [4] and Zugenmaier [6]. In addition to these results which apply to the particular case of sidechain liquid crystalline polymers, it is also useful to recall some classical considerations about the stereochemistry of polymer chains in general [16].

2.1 RÓle OF THE FLEXIBLE SPACER. - In the amorphous state, polymer chains usually tend to adopt a three-dimensional random-coil conformation; on the other hand, rod-like mesogens tend to orient themselves parallel to one another to form nematic mesophases and, eventually, to pack in superposed layers to produce smectic liquid crystals. In side-chain liquid crystalline polymers, these tendencies are thought to conflict with each other. Consequently, the presence of flexible spacers in the macromolecules is thought to be essential for the formation of liquid crystals, as it would induce mechanical decoupling of the mesogenic pendant groups from the polymer backbone, hence allowing mesogenic groups to order freely [3].

Concerning the presence of the flexible spacer, we believe that it can explain, to a large extent, the occurrence of smectic phases, but that it is not able to justify the formation of nematic phases. First, from a thermodynamic point of view, it has been established [17-18] that classical polymers have very low solubility in low-molecular weight mesomorphic phases. Now, binding mesogenic groups to polymer molecules through flexible spacers suppresses macroscopic phase separation, but this, of course, can by no means be taken as an indication that the two species have become compatible with one another. The same situation is encountered with soap-like substances [19] (including rod-like smectogens [20]), where the two incompatible constituent parts of the molecules persist in repelling one another despite their covalent linkage, giving rise to the well-known amphiphilic character. As a result, there is local segregation of the two incompatible moieties of the soap molecules with creation of an interface and formation of smectic-like ordering. Applied to sidechain polymers, this means that, even if there were complete mechanical decoupling of the pendant groups from the backbone, the incompatibility of these species would favor the formation of interfaces, thus of smectic phases, and correlatively, that it would disfavour the absence of positional correlations among the molecules, thus the formation of nematic phases.

Second, from another thermodynamic argument, it is well-known that, in lyotropic systems of rod-like macromolecules, nematic liquid crystals are only obtained at solvent concentrations which increase with the aspect ratio of the rods [21, 22]. This explains why low-molecular weight nematic phases, for which the aspect ratio of the rods (typically, about 3) is close to unity, are destroyed upon addition of very little solvent (typically, less than a few percent) [23]. In side-chain polymers, the corresponding solvent content is considerable, as it is the sum of the polymer backbone and spacer concentrations. This leads one to wonder whether sidechain polymers are able to produce classical nematic phases, with the pendant groups oriented in a common direction.

Third, from a purely geometrical point of view, one can easily conceive the formation of smectic mesophases with side-chain liquid crystalline polymers, but not of classical nematic ones. Indeed, in the case of smectics, the mesogenic pendant groups, which are next to each other along the polymer backbone, can very well be in lateral register and arrange themselves in layers. The polymer chains should then be confined to just lying in between the layers. This will be discussed further below. In the case of nematics on the other hand, the mesogenic groups should, by definition, be parallel but positionally uncorrelated, especially in the direction of their elongational axis. With short-spacer sidechain polymers where nematics are reported to occur, this is impossible since adjacent pendant groups are attached to the backbone at distances which are small compared to their length. Furthermore, the polymer backbone, whose lateral bulkiness is at least equal to that of the pendant groups, can hardly clear its path through the parallel pendant groups to approach a random coil conformation without greatly disturbing the nematic ordering of the mesogenic moieties.

2.2 THE HERRING-BONE CONFIGURATION. - Regarding the configuration of the pendant groups with respect to the polymer main chain, these are typically described as emerging alternately from opposite sides of the polymer backbone in a sort of herringbone arrangement. The smectic layers consist of such ribbon-like polymer molecules either packed side by side to give double layers of pendant groups, or intercalated to give single layers [4, 6, 24] (Fig. 1).

From a geometrical point of view, we believe this configuration is indeed quite plausible as it takes into account the lateral thickness of the pendant groups (about $5 \AA$ ) compared to their chemical repeat distance (about $2.5 \AA$ ) along the polymer backbone. The herring-bone arrangement seems to reasonably satisfy the requirement for the pendant groups to pack efficiently, with a lateral approach distance corresponding to their size, and without undue strain of the polymer main chain. One is, however, drawn to ask whether this is the only arrangement possible for the pendant groups to fill the space, and furthermore, how this arrangement is 


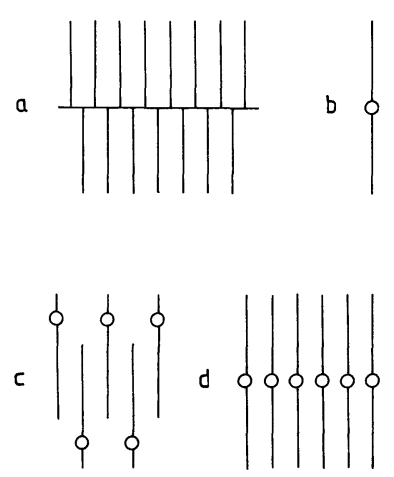

Fig. 1. - The herring-bone model : a, side-view of a single polymer chain $; b$, end-on view of a single polymer chain ; c, intercalated single-layered packing ; d, doublelayered packing.

affected by the stereochemical configuration of the backbone.

\subsection{STEREOCHEMISTRY OF POLYMERS ( $\left.{ }^{1}\right)$. -} Through systematic X-ray diffraction work, Natta and coworkers [16] came to the conclusion that in crystalline isotactic vinyl polymers, the conformation of the polymer chains is helical, as shown in figure 2 . This is consistent with the fact that successive monomer units have the same steric configuration, and can therefore be deduced from one another by a simple rotation and translation, the twist of the helix being related to the bulkiness of the side groups, $\mathrm{X}$, and to their interaction. As for the crystalline syndiotactic polymers, the conclusion was that the polymer chains have a conformation which presents a glide-plane symmetry, as shown in figure 3 . This is consistent with the fact that successive monomer units have opposite steric configurations, and need therefore to be deduced from one another through symmetry operations involving the presence of centers of inversion.

As the polymers used in this study have poly(methacrylate) backbones, it is useful to recall tacticity and conformational results concerning poly(methyl methacrylate), PMMA, which is the most extensively studied member of the series, and perhaps the simplest representative of the poly(methacrylate) backbone :

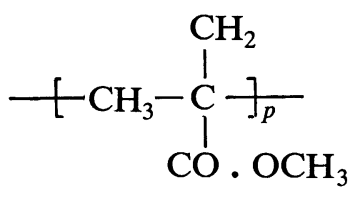

Highly isotactic PMMA is extremely difficult to thermally crystallize in the bulk [27]. It has nonetheless been crystallized from solution [28-32]. Using Xray diffraction, it was shown to form a structure with a double-strand helix, each strand being a $10_{1}$ helix [33]. Pure, highly syndiotactic PMMA is impossible to thermally crystallize in the bulk as was the case for isotactic PMMA. It has, however, also been crystallized in the presence of a solvent [34]. By X-ray diffraction, the structure was found to be complex, involving a local chain conformation near the alltrans conformation inferred from conformational energy calculations [35], that is similar to the chain conformation shown in figure 3. Although PMMA

(1) In head-to-tail polymerized vinyl polymers, $\left[\mathrm{CH}_{2}-\mathrm{C}^{*} \mathrm{HX}\right\}_{p}$ asymmetric carbon atoms $\left(\mathrm{C}^{*}\right)$ are alternated with methylenic groups along the polymer backbone. From a stereochemical point of view, the atoms covalently bonded to $\mathrm{C}^{*}$ give rise to two elementary configurational units, one being the mirror image of the other $(\mathrm{R}=$ rectus, $\mathrm{S}=$ sinister $)$. The occurrence of a given configuration around each tetrahedral $C^{*}$ atom is determined once and for all during the polymerization process. The tacticity of the polymer chain is defined by the distribution of these two enantiomeric configurations along the polymer chain [16]. It is of importance to note the difference between configuration (tacticity) and conformation of polymer chains (random coil). The first describes the local stereochemical arrangement of atoms within the chain, whereas the second refers to the global shape of the polymer chain and is determined by internal rotations around the backbone $\mathrm{C}-\mathrm{C}^{*}$ covalent bounds. Though the tacticity can affect the conformation of the polymer chain through the interactions between atoms, changes in conformation never affect the tacticity.

A group of two successive configurational units in the polymer chain are said to form a meso dyad $(\mathrm{m})$ if the configuration of the backbone $\mathrm{C}^{*}$ atoms involved is the same $(m=R R$ or $\mathrm{SS})$ or a racemic dyad ( $\mathrm{r}$ ) if the configuration is different ( $r=R S$ or SR). Just as the term dyad refers to dimers in the polymer chain, the term triad refers to trimers. There are three types of triads possible : the isotactic triads (i) if the three successive $\mathrm{C}^{*}$ atoms are in the same RRR or SSS configuration (mm); the syndiotactic triads (s) if the successive $\mathrm{C}^{*}$ atoms are in an alternating RSR or SRS configuration (rr); and the heterotactic triads $(\mathrm{h})$ if the $\mathrm{C}^{*}$ atoms are in a RRS, SRR, RSS, or SSR configuration ( $\mathrm{mr}$ or $\mathrm{rm})$. The tacticity of a given polymer is defined by the relative proportion of $i, s$, and $h$ triads measured by N.M.R. [25]. For example, ideally isotactic and syndiotactic polymers contain $100 \% \mathrm{i}$ and $\mathrm{s}$ triads respectively; ideally atactic polymers contain equal amounts of elementary $\mathbf{R}$ and $\mathrm{S}$ configurational units randomly distributed along the polymer chain, corresponding to 50,25 , and $25 \%$ of $\mathrm{h}, \mathrm{i}$, and s triads, respectively. It should be noted, however, that in common use, polymers are said to be isotactic or syndiotactic when they are simply rich in $\mathrm{i}$ or $\mathrm{s}$ triads, otherwise they are said to be atactic.

In most polymerization processes of vinylic monomers, neither ideally isotactic or syndiotactic, nor ideally atactic polymers are formed. As a rule, because of their geometrical irregularity these polymers are unable to crystallize, remaining in the amorphous state. However, there are now classical chemical synthetic routes to synthesize vinyl polymers with high isotactic or syndiotactic triad contents [26]. These polymers often crystallize due to their regular character. 


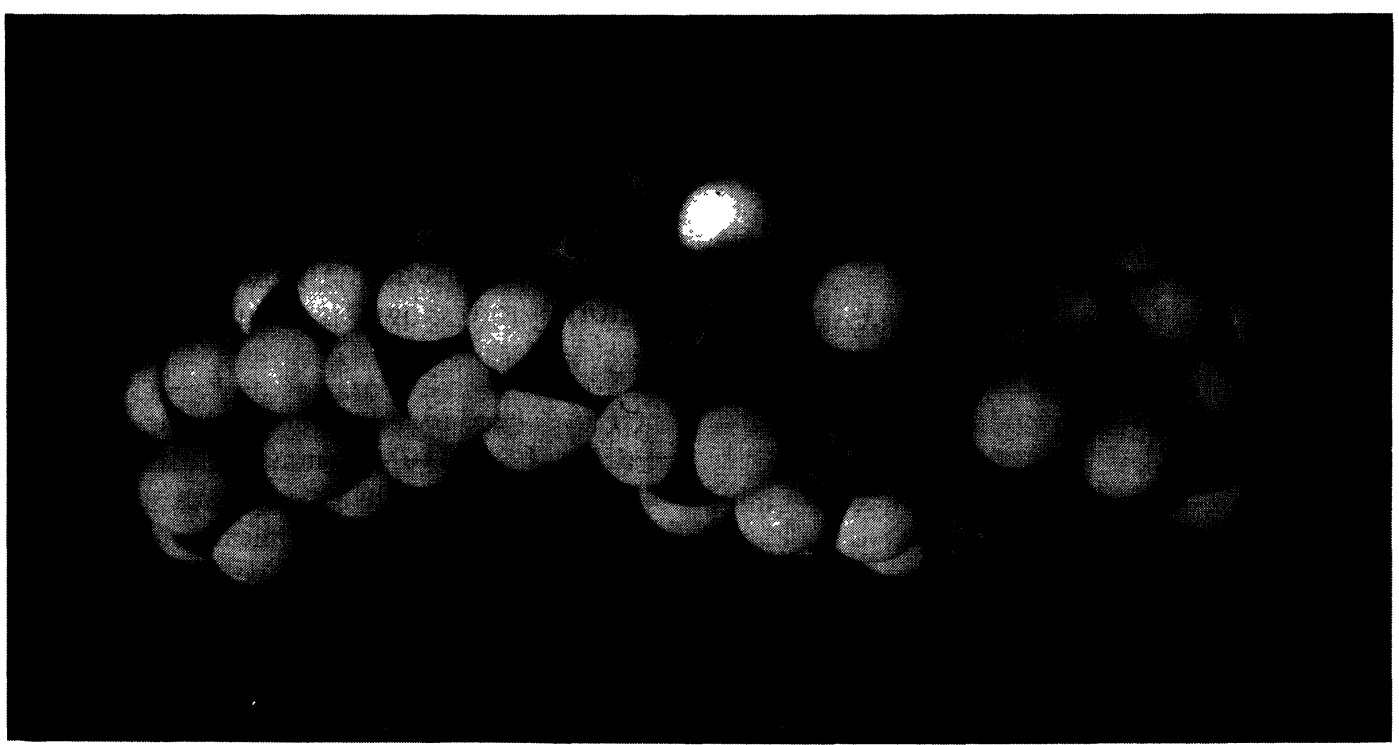

Fig. 2. - Molecular CPK model showing the helical chain configuration of isotactic poly(methylmethacrylate).

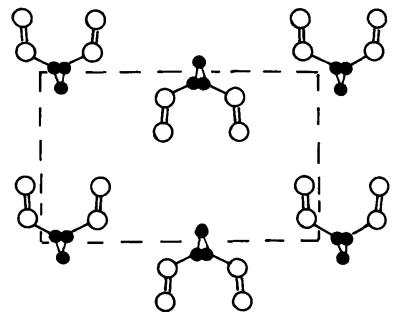

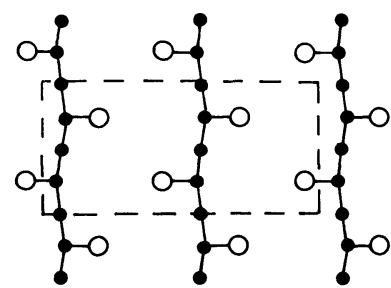

Fig. 3. - The structure of syndiotactic 1, 2-polybutadiene $\left.-\mathrm{CH}_{2}-\mathrm{CH}\left(\mathrm{CH}=\mathrm{CH}_{2}\right)\right]_{p}$. Filled circles represent the backbone carbon atoms, and empty circles represent carbon atoms in the pendant vinyl group (adapted from Ref. [16] with permission of editor).

structural behaviour is rather complicated, it nevertheless generally follows the behaviour of other vinyl polymers : isotactic PMMA has a helical conformation and syndiotactic PMMA has a local conformation close to the glide-plane type.

Normal free-radically polymerized PMMA has about $66 \%$ syndiotactic triads [36, 37] and is amorphous. Wide-angle X-ray scattering analysis of an amorphous PMMA sample with similar tacticity has shown that, in spite of the amorphous state of the material, the conformation of the backbone is regular on a local scale of about 8 to 10 monomer units [38]. This conformation (Fig. 4) is near the alltrans energy-minimum conformation proposed in reference [35]. With both the methyl and ester groups on the same side of the main chain, a small curvature is induced in the backbone due to the steric hindrance of the first atoms next to the main chain. This curvature is sufficient to hinder a complete filling of the space in a crystalline way, and thus leads to an amorphous material.

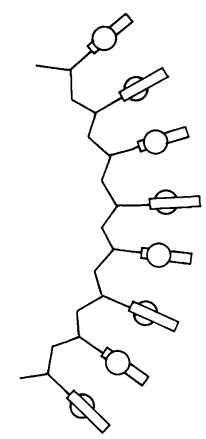

Fig. 4. - Schematic view of the local syndiotactic conformation of poly(methylmethacrylate) taken from reference [38]. Zig-zag line represents the polymer backbone, empty circles represent the methyl groups and rectangles the ester groups.

\section{Experimental.}

3.1 MATERIALS. - The poly(methacrylates) used in the present work were all free radically polymerized [10]. They are indicated in the following by the abbreviation PM- $n$; they have 4'-methoxybiphenylyl mesogenic pendant groups and oligo(ethylene oxide) spacers of various lengths : 


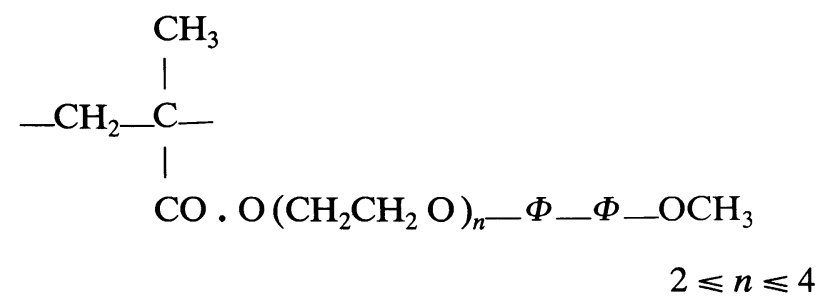

The physico-chemical properties of the polymers were studied by light scattering and size exclusion gel permeation chromatography [11]. Their tacticity was determined by nuclear magnetic resonance [10]. Experimental data on molecular weights, polydispersities, and tacticities are shown in table I.

Table I. - Molecular weights, polydispersities, and tacticities of the polymers studied.

\begin{tabular}{ccrrrrr}
\hline Ref. & Sample & $10^{-3} M_{\mathrm{w}}$ & $M_{\mathrm{w}} / M_{n}$ & \multicolumn{3}{c}{$\begin{array}{c}\text { Tacticity } \\
\text { i } \% \text { h \% s }\end{array}$} \\
\hline PM-2 & RP1 & 65.0 & 2.8 & 12 & 34 & 54 \\
PM-3 & RP6 & 45.9 & 2.6 & 11 & 35 & 54 \\
PM-4 & RP49 & 183.0 & 2.7 & 11 & 34 & 55 \\
\hline
\end{tabular}

The thermotropic polymorphism of the polymers, including glass transitions, was studied by differential scanning calorimetry (DSC), optical microscopy, and dilatometry [12]. Using previous structural observations [13-15] and results to be discussed below, this polymorphism can be described as follows :

$$
\begin{array}{ll}
\text { PM-2 (RP1) : } & \mathrm{S}_{\mathrm{E}, \text { glass }} \stackrel{94^{\circ} \mathrm{C}}{\longleftrightarrow} \mathrm{S}_{\mathrm{E}} \stackrel{148^{\circ} \mathrm{C}}{\longleftrightarrow} \mathrm{I} \\
\text { PM-3 (RP6) : } & \mathrm{S}_{\mathrm{E}, \text { glass }} \stackrel{49^{\circ} \mathrm{C}}{\longleftrightarrow} \mathrm{S}_{\mathrm{E}} \stackrel{107^{\circ} \mathrm{C}}{\longleftrightarrow} \mathrm{I} \\
\text { PM-4 (RP49) : } & \mathrm{S}_{\mathrm{E}, \text { glass }} \stackrel{38^{\circ} \mathrm{C}}{\longleftrightarrow} \mathrm{S}_{\mathrm{E}} \stackrel{0^{\circ} \mathrm{C}}{\longrightarrow} \mathrm{I} .
\end{array}
$$

3.2 X-RAY DIFFRACTION. - The X-ray diffraction study of the PM- $n$ polymers was carried out on unoriented powder samples and on fibers drawn from the polymer melt. Attempts were also made to magnetically orient specimens. Diffraction patterns were photographically recorded with three types of cameras. The first was a Guinier focusing camera equipped with a bent quartz monochromator and using $\mathrm{Cu}-\mathrm{K} \alpha_{1}$ radiation from a PW1009 Philips generator. The second was a Searle camera with toroidal optics using $\mathrm{Ni}$-filtered copper radiation from an Elliott GX20 rotating anode generator. Both of these cameras were used with unoriented powder samples and had electronically controlled ovens allowing the heating of the samples in the temperature range from 25 to $300{ }^{\circ} \mathrm{C}$ within an accuracy of $1^{\circ} \mathrm{C}$.

The third camera was a custom-made camera equipped with two bent gold-plated glass mirrors crossed to give a point- focused direct beam. It used Ni-filtered copper radiation from an Elliott GX20 generator. With a sample to film distance of $100 \mathrm{~mm}$, it was aligned to allow registration of Bragg reflections in the spacing range from 3 to $100 \AA$ with a resolution of about $1000 \AA$. Fiber samples were mounted in a modified Mettler FP52 heating stage whose temperature was electronically controlled within $0.1{ }^{\circ} \mathrm{C}$.

\section{Results and discussion.}

4.1 X-RAY DIAGRAMS. - X-ray diffraction patterns were registered for powder samples of PM-2, PM-3, and PM-4 at a variety of temperatures between room temperature and the clearing point. Above $T_{\mathrm{g}}$, structural equilibrium was reached fairly rapidly and annealing did not produce significant changes in the patterns. Likewise, temperature did not seem to appreciably affect the structural parameters. The patterns (Fig. 5) all consisted of a series of three equidistant sharp reflections in the small-angle region, corresponding to lamellar spacings of 23.0, 26.9, and $29.8 \AA$ for PM-2, PM-3, and PM-4 respectively, and of three rather sharp reflections in the wide-angle region corresponding to spacings of 4.54 , 4.01 , and $3.23 \AA$. The intensity sequence of the small-angle reflections was very weak, medium, and very weak respectively, with a tendency for the third harmonic to decrease in intensity as the spacer length increases. The wide-angle intensities were strong, medium, and weak respectively.

In order to get more information about the structure of the polymers, much effort was given to obtaining oriented samples. Along this line, orientation by slow cooling from the melt in magnetic fields up to $4.2 \mathrm{~T}$ turned out to be unsuccessful, presumably due to the absence of nematic phases or, perhaps, also due to the rather high viscosity of the medium. However, significant orientation was ob-
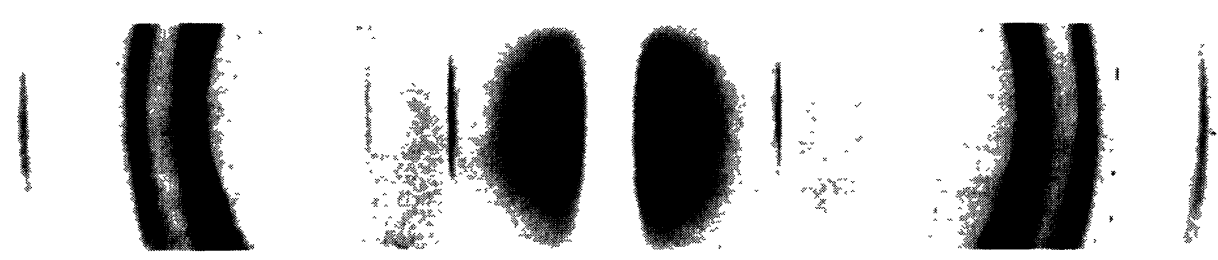

Fig. 5. - Guinier X-ray diffraction powder pattern of PM-2. 
tained by pulling fibers from the melt. The X-ray patterns registered show that the small-angle reflections present little arcing and are equatorial with respect to the draw direction, while the wide-angle reflections are highly arced, with little reinforcement in the meridian direction (Fig. 6). Bragg spacings and intensities corresponded to those determined from the powder patterns.

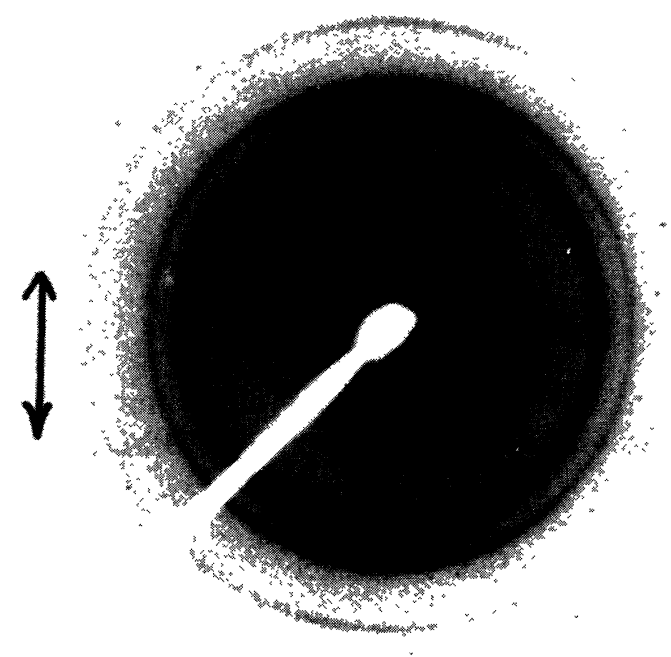

Fig. 6. - Fiber X-ray diffraction diagram of PM-4. Arrow indicates the draw direction.

4.2 SMECTIC ORDERING OF THE PENDANT GROUPS. This section is mainly concerned with the ordering of the polymer mesogenic groups alone ; the ordering of the backbone itself will be considered more extensively in the next sections. The X-ray diagrams registered closely resemble those obtained with lowmolecular weight rod-like smectogens. They are indicative of an ordered smectic structure, in agreement with the microscopic observations of the textures [12-14].

The thickness of the smectic layers measured varies linearly with the spacer length (Fig. 7). The slope of the corresponding line gives the projected length of one ethylene oxide repeat unit in the spacer along the layer normal, while the Y-intercept ( $d$ for $n=0$ ) gives the thickness of the polymer backbone and methoxybiphenylyl sublayers superposed. The $d$ spacings for the polymers corresponded exactly to the length of one monomeric unit in the fully extended conformation. This suggests that the smectic structure corresponds to single layers of pendant mesogenic groups oriented perpendicular to the layers.

The three wide-angle $\mathrm{X}$-ray reflections are identical for the three polymers and are therefore inde-

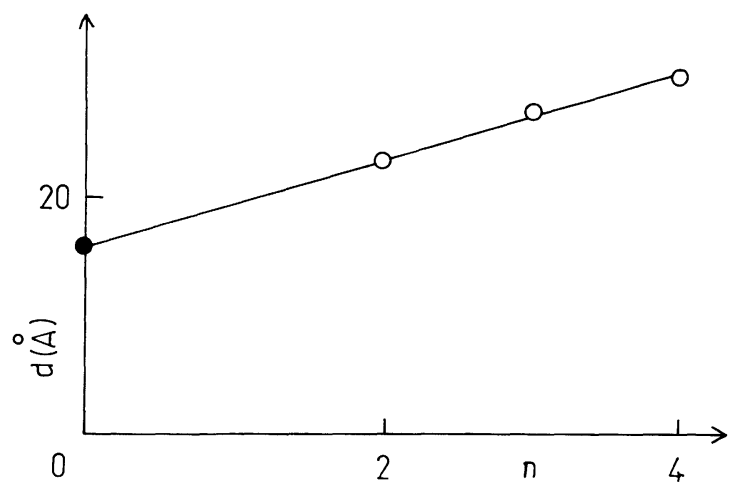

Fig. 7. - Layer thickness, $d$, as a function of the number, $n$, of ethylene-oxide repeat units in the spacer. Filled circle represents the layer thickness of PM-0 (from Ref. [15]).

pendent of the length of the pendant groups. They can be interpreted as corresponding to the twodimensional lateral packing of the mesogenic moieties. Their angular position in the X-ray patterns and their intensity sequence are in close agreement with the observations by Doucet et al. [39] on the smectic E phase of low-molecular weight mesogens. The smectic E structure described involves a lateral packing of the molecules within the layers according to a two-dimensional pseudo-centered rectangular lattice. The wide-angle reflections of the three polymers can be indexed as 110, 200, and 210 reflections of a two-dimensional rectangular lattice with the cell parameters $a=8.02 \AA$ and $b=5.54 \AA$ (Fig. 8). With two pendant groups per unit cell, this gives a molecular area per pendant group of $S=a * b / 2=22.2 \AA^{2}$. This molecular area corresponds very well with the value of $21.8 \AA^{2}$ deduced from the cell parameters given by Doucet for the low-molecular weight smectic E mesogens.

Concerning the stacking of the smectic layers, it is worth noting that, in classical smectic E mesophases, the ordered smectic layers are positionally correlated in the three-dimensional space. This gives rise to a true three-dimensional orthorhombic crystal. The

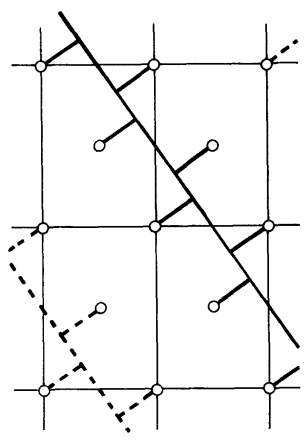

Fig. 8. - Two-dimensional pseudo-centered rectangular lattice. Open circles represent the pendant groups seen end-on and thick lines represent schematically the polymer backbone and the flexible spacer; dashed lines indicate backbone and spacers from layer below. 
three-dimensional correlation is manifested in the $\mathrm{X}$ ray patterns by the presence of $h k l$ reflections, particularly the 111,201 , and 211 reflections, which appear as satellites of the 110,200 , and 210 ones. In the case of the polymers studied in this section, both the powder and fiber X-ray patterns show no evidence of $h k l$ reflections. It seems, therefore, that three-dimensional correlations between the layers of pendant groups are either absent here or extremely weak.

4.3 CONFORMATION OF THE POLYMER CHAINS. The smectic structure of the polymers has so far been interpreted in terms of layering and lateral packing of the pendant groups alone. Before describing the way the polymer molecules as a whole are arranged in the smectic layers, it is necessary to consider the conformation of the backbone and its relation to the pendant groups.

To deal with the backbone, the most obvious route is first to use the classical description of smectic side-chain liquid crystals [6], summarized in section 2.2 and shown in figure 1. The polymer backbone and the attached pendant groups are described to adopt a sort of planar herring-bone configuration. The smectic layers are formed by these planar ribbon-like herring-bone polymer units packed side by side. Depending on whether the ribbons are in lateral register (Fig. 1d), or intercalated, partially overlapping (Fig. 1c), the stacking period of the lamellar system should correspond to the length of either two or one fully extended pendant groups.

Experimental evidence obtained in the present work with the PM-2, PM-3, and PM-4 polymers is not in favour of such a classical description. As discussed in the previous section, the thickness of the smectic layers measured corresponds to the length of one extended pendant group, thus precluding the double-layer model illustrated in figure $1 \mathrm{~d}$. On the other hand, the intercalated herring-bone model shown in figure $1 \mathrm{c}$ is unacceptable as well. Indeed, it should give rise to strong three-dimensional correlations between pendant-group sublayers. This is contrary to the observed lack of $h k l$ Bragg reflections presented above. Intercalation should also make it very difficult for the smectic layers to slide over one another. This is contrary to the observation under a microscope that the smectic $E$ phase could be sheared, and to the orientation obtained in X-ray fiber diagrams corresponding to the smectic layers all oriented by shear parallel to the draw direction.

Another true single-layer model can be envisaged, however, which is in agreement with the observed single-layer arrangement of the pendant groups. This model has already been proposed for alkyl sidechain polymers $[40,41]$ but was immediately rejected as being unlikely. Here the pendant groups all hang on the same side of the polymer backbone. As they are too bulky to arrange in a single row, the pendant groups are laterally displaced in an alternate fashion to form a staggered double row as shown in figure 9. This allows for the pendant groups to stand at an approach distance of about $5 \AA$, compatible with their steric hindrance and also with their chemical repeat distancè along the backbone.

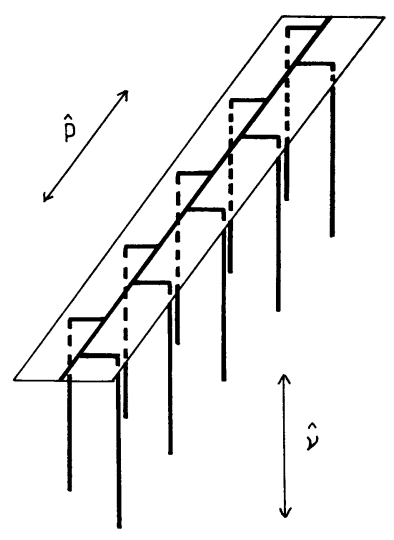

Fig. 9. - Schematic view of the ribbon-like configuration of the PM- $n$ polymers.

Before going further, it is necessary to ask whether one is allowed to place the pendant groups either on the same side of the backbone or in the herring-bone configuration at liberty, without considering the stereochemical implications of the model first. In this context, it is of particular importance to consider the role of the backbone tacticity. Indeed, as discussed in section 2.3 , tacticity plays a decisive part in the conformation and the ordering of polymer chains in the solid state : isotactic polymers usually form helices, while syndiotactic ones lead as a rule to glide planes.

In order to analyse the effect of tacticity, the CPK molecular models were constructed for the PM-2 polymer with various tacticities. For an isotactic backbone, the polymer was found to form a helical conformation as was expected. For the true atactic PM-2, the polymer was found to adopt a very irregular conformation. In both cases, in spite of the presence of flexible spacers (at least for the spacer lengths considered in this work), the pendant groups could not be oriented parallel to one another as required for smectic layering. As for the syndiotactic material, the CPK molecular models gave a very regular conformation with the pendant groups parallel to one another and on the same side of the backbone as needed for the formation of singlelayered smectics (Fig. 10). It should be noted that this conformation is in agreement with that proposed by Windle [38] for amorphous PMMA. 


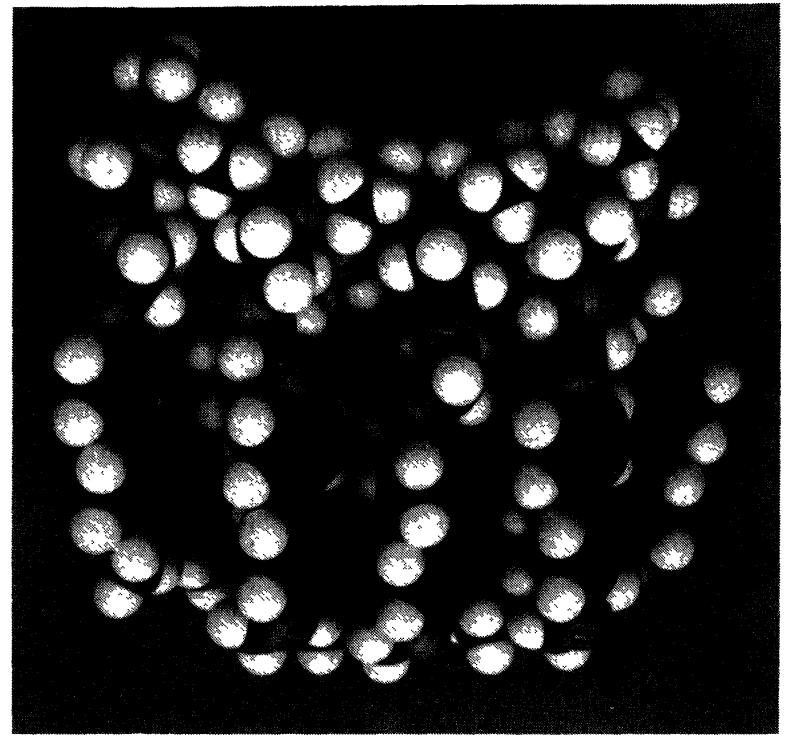

Fig. 10. - CPK molecular model of syndiotactic PM-2 polymer.

As with PMMA [38], the CPK molecular models of PM-2 showed a small curvature of the polymer backbone, with both the $\alpha$-methyl and pendant groups on the convex side. The curvature is connected with steric hindrance effects, largely coming from the first atoms next to the main chain, and results in an appreciable splay distortion of the pendant groups. Small increases in the bond angles between successive backbone carbon atoms spread slightly the distance between pendant groups, thus relieving the steric hindrance and decreasing the splay distortion. Using energy data from conformational analysis of PMMA [35] and from splay elastic distortion calculations by continuum theory [42], it can be seen that such angular distortions to straighten out the backbone are entirely possible. In the case of PMMA the straightening out of the backbone does not occur, presumably because of the lack of elastically interacting rigid rod-like pendant groups.

The above analysis concerns the conformation of pure syndiotactic materials. The PM-2, PM-3, and PM-4 polymers have only about $55 \%$ syndiotactic triads, that is about $75 \%$ racemic dyads. On the average, this means that in these polymers, supposed to be globally syndiotactic, there is one meso defect every four dyads. To check how much the presence of meso defects changes the conformation, the CPK models of eight successive PM-2 monomeric units were constructed in a syndiotactic structure with one meso defect every two, three, and four dyads respectively. It was clear that defect levels of less than one meso per three dyads led to conformations (Fig. 11) still very regular and similar to those obtained with pure syndiotactic polymers. Significant departures from the shape of pure syndiotactic

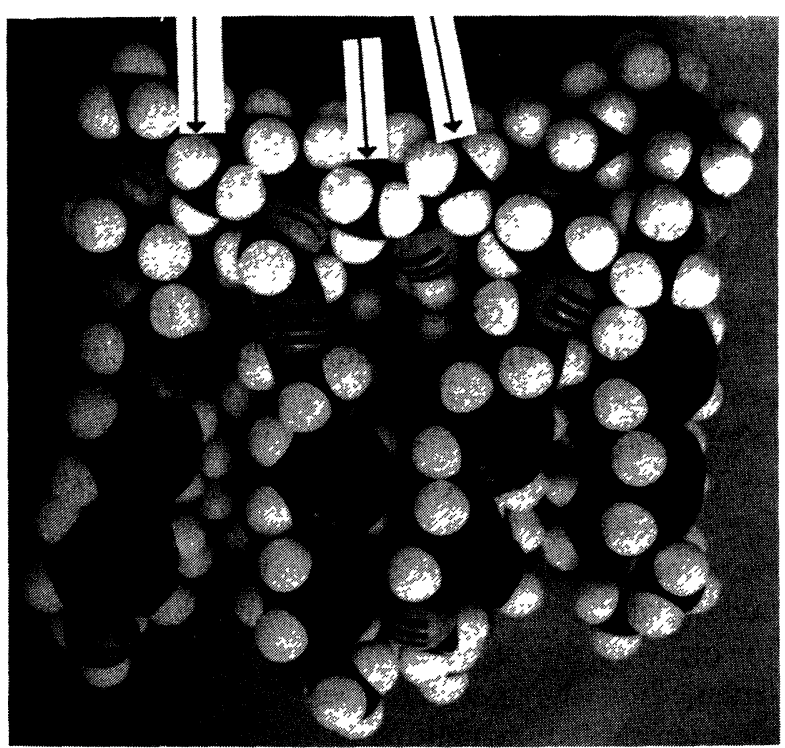

Fig. 11. - CPK molecular model of syndiotactic PM-2 polymer with one meso defect (shown by arrows) every four dyads.

material occurred at higher defect levels. The PM- $n$ polymers considered in the present work are therefore perfectly interpretable in terms of the syndiotactic model.

To this point, the pendant groups have been described to hang parallel to each other on the same side of the polymer backbone, and the main chain conformation has been shown to be easily taken as that of the purely syndiotactic polymer backbone. This leads to an overall ribbon-like shape for the polymer chain as shown in figure 9 . In other words, the PM- $n$ polymer molecules form ribbons, the ideal length of which is related to the degree of polymerization (from about 500 to $1000 \AA$ ). The width of a ribbon corresponds to the length of one extended monomeric unit (from about 15 to $30 \AA$ ), and its thickness to roughly twice the lateral bulkiness of the pendant groups (about 8 to $9 \AA$ ). It is these ribbons that now have to be considered as being the structural units of the smectic layers.

4.4 ORDERING OF THE POLYMER CHAINS. - Combining the above information, the structural model presented in figure 12 was proposed for the PM-2, PM-3, and PM-4 polymers. The ribbon-like polymer chains are packed side by side with the polymer backbone parallel to the smectic layers and the mesogenic groups perpendicular to them. The rigid biphenylyl groups are in lateral register with one another forming the two-dimensional pseudo-rectangular lattice shown in figure 8 . The polymer backbones are also in register with one another, lying in planes located between the biphenylyl sublayers, in the center of the disordered sublayer formed by the ethylene oxide spacers and the 


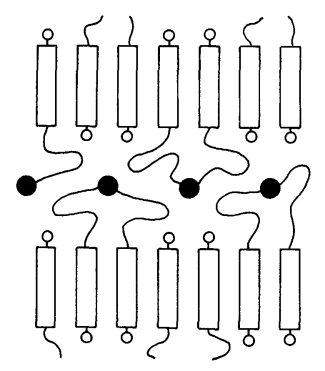

Fig. 12. - Schematic representation of the single layered smectic structure of the PM- $n$ polymers. Filled circles represent the polymer backbones viewed end-on, wavy lines represent the flexible spacers, rectangles represent the mesogenic pendant groups, and small open circles represent the terminal methoxy groups.

methoxy terminal groups. The ribbons within a layer are oriented in an up and down fashion, analogous to a paraelectric or antiferroelectric arrangement. This model is thought to be the most plausible explanation of the experimental data obtained in the present work and to be in agreement with common knowledge of liquid crystals and considerations that will be discussed below.

The up and down orientation of the pendant groups of adjacent ribbons in a layer is similar to that seen in the case of lyotropic smectic B mesophases of polysoaps [43] where the ionic polymer chains with pendant aliphatic moieties also have a ribbon-like shape. Such an up and down orientation is quite common in the liquid crystalline field for anisotropic rod-like molecules. The only exceptions known to this rule are the special cases of double-layered smectics (where the molecules are oriented in the same direction in each sublayer) [20] and chiral smectics C [44]. The question now is whether the up and down orientation of the ribbons is random or alternating. By simple density arguments, it is clear that the orientation should be mostly of an alternating nature. Indeed, from dilatometry experiments [12], the flexible ethylene oxide spacers have been shown to be disordered. The random conformation of the flexible spacers implies large molecular areas and greatly disfavors the same orientation for adjacent ribbons which would involve tight packing of the spacer portions of the ribbons and small molecular areas. If the ribbons were oriented in the same direction, even if only on a local scale, the flexible spacers would emerge on the same side of the biphenylyl sublayer and would adopt a molecular area comparable to that, about $22 \AA^{2}$, of the biphenylyl groups themselves. Obviously, this value is too small for the flexible spacers to freely adopt a random conformation. In the model proposed in the present work with an antiparallel orientation of the ribbons, the molecular area of the spacers is instead twice as large.
The location of biphenylyl groups, the polymer backbones, and the flexible spacers in three distinct sublayers superposed to form the smectic structure is in agreement with the requirements imposed by the amphiphilic character of the molecules [20]. Indeed, in the absence of specific attractive forces (dipoledipole interactions, hydrogen bonding...), these three species tend to be incompatible with one another from an enthalpic point of view (Van der Waals interactions), resulting in a tendency towards local segregation. In addition, the segregation of the biphenylyl groups in distinct sublayers is in perfect agreement with the fact that these groups are found to be in good register with one another, and arranged according to a well defined two-dimensional lattice.

A further argument in favour of the structural model proposed comes from the analysis of the intensities of the small-angle X-ray reflections. As already stated in section 4.1 , the second harmonic is significantly stronger than the first and the third ones. This is consistent with a layered structure presenting strong electron density correlations along the normal to the layers with a pseudo-period of half a layer thickness. In order to verify whether such is the case with the structural model shown in figure 12 , the one-dimensional electron density distribution along the normal to the layers was estimated. For this purpose, the projected lengths (to the layer normal) of the biphenylyl, the backbone, the terminal methoxy, and the spacer were estimated for the PM-2 polymer using X-ray diffraction data (Fig. 7) and CPK molecular models : these lengths were found to be $8.8,4.8,2.5$, and $6.9 \AA$ respectively. From the known number of electrons in each group the linear electron density distribution shown in figure 13 was deduced. It is seen that the electron density does indeed have a pseudo-period of half the layer thickness, in agreement with the observed Xray intensity data and the location of the polymer backbones exactly between the biphenyl sublayers (Fig. 12).

One must now consider the different ways of arranging the polymer backbones with respect to the

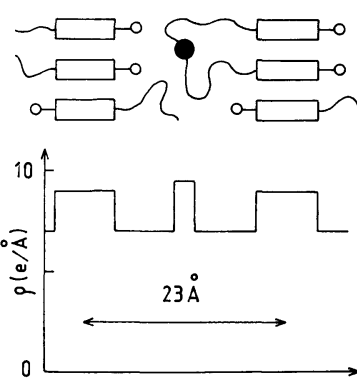

Fig. 13. - One-dimensional electron density distribution along the normal to the layers. 
rectangular two-dimensional packing of the pendant groups and also with respect to each other. From the rectangular packing shown in figure 8 , it can be seen that each pendant biphenylyl group has first neighbours located at $4.87 \AA$ in the [110] direction, then further neighbours at 5.54 and $8.02 \AA$ in the $[010]$ and $[100]$ directions, respectively. Taking into account the chemical repeat distance between successive pendant groups along the polymer chain, $2.54 \AA$, corresponding to a maximum effective distance of about $5 \AA$ between two successive homologous pendant groups in a given row of the ribbon (as shown in Fig. 9), it is clear that the backbone cannot run in the [100] or [010] directions. The only possible path the polymer backbone can follow is, therefore, the [110] direction of the diagonals of the unit cell.

With the above knowledge, one can see that, in principle, there are four allowed diagonal directions, $[ \pm 1, \pm 1,0]$ that the polymer backbone can follow in its sublayer. One might imagine, therefore, that the polymer backbones can adopt a broken-line configuration in a sort of two-dimensional random walk. This supposes that the polymer chains make abrupt direction changes of $2 * \tan ^{-1}(a / b)$ or $\simeq 111^{\circ}$, and $2 * \tan ^{-1}(b / a) \simeq 69^{\circ}$, which is highly unlikely considering the compactness of the CPK models for the PM- $n$ polymers (Fig. 10) and the rigidity of the polymer backbone. This leads to the conclusion that the polymer backbones are rectilinear and run parallel to one another over relatively large distances. Such a conclusion is all the more satisfactory since, with the backbone in the [110] direction, the lateral distance between neighbouring polymer ribbons is equal to

$$
2 a b / \sqrt{\left(a^{2}+b^{2}\right)} \simeq 9.1 \AA,
$$

in agreement with the thickness of the ribbons estimated from the CPK molecular model.

It is now worth discussing in some detail the correlations between successive superposed layers. From the X-ray patterns presented in section 4.1, and as stated in section 4.2 dealing with the smectic ordering of the pendant groups, it is clear that there are no long-range three-dimensional positional correlations between the layers. The question remains of whether there is orientational correlation between them. To answer this question, it is important to recall the following features. First, the pendant groups are intercalated in the aromatic sublayers due to their up and down orientation. Second, the polymer backbones are arranged parallel to one another in the polymer sublayers due to their rectilinearity and rigidity. The obvious conclusion is that there should be rather strong orientational correlations between smectic layers. This suggests a hexatic-type ordering where there is long-range three-dimensional orientational correlation and no positional correlation between layers [45]. The rather sharp reflections obtained in the wide-angle region indicate that the positional correlation of the pendant groups within a biphenylyl sublayer is rather longrange. From the point of view of the range of orientational and positional correlations, the smectic structure of these polymers would therefore be reminiscent of a smectic I structure [46] (but with no tilt of the molecules). In smectic I phases, the preferred orientation is given by the tilt of the molecules towards the short edge of the rectangular lattice, whereas in the PM- $n$ polymers it is given by the diagonal orientation of the polymer backbones.

To finish this section, it should be noted that the structural model presented here is a refinement of one given in a previous preliminary note [14] dealing with the isolated case of PM-2. With a small shift of the ribbons parallel to the direction of the pendant groups, it takes into account the amphiphilic character of the smectogenic polymer and provides a better interpretation of the small-angle $\mathrm{X}$-ray intensity sequence.

4.5 Remarks. - The above analysis suggests the following remarks on the macroscopic orientation of the PM-2, PM-3, and PM-4 polymers obtained by drawing fibers from the melt. In the X-ray fiber diagrams registered (see Sect. 4.1), it is apparent that the smectic layers are consistently oriented parallel to the draw direction. The very large arcing of the reflections in the wide-angle region suggests that, on the contrary, the two-dimensional rectangular lattice and the polymer backbones are not substantially oriented on a macroscopic scale along the draw direction. The interpretation is that orientation is obtained primarily by shearing of the smectic layers and not by pulling of the polymer molecules. Such shearing cannot be done without disrupting the sublayers, presumably involving unlocking of polymer backbones within their sublayer. The rheological element involved in the shearing process seems, therefore, to be formed by the smectic layers rather than by the polymer chains themselves. This is consistent with the fact that long annealing is necessary to recover the quality of the structure after drawing.

\section{Conclusion.}

In this paper, a systematic X-ray diffraction study of a homologous series of side-chain liquid crystalline poly(methacrylates) is presented. To interpret the experimental results in a methodical manner, it was felt useful to introduce some basic concepts from different fields concerning both polymer and liquid crystal science. It was thus possible to understand the structure of the PM- $n$ polymers studied in the present work and to appreciate the rôle of the 
polymer backbone conformation on the ordering of the pendant groups.

Experimental results pointed to a single-layered, ordered smectic structure in which the superposed smectic layers were positionally uncorrelated with one another. This kind of structure was difficult to understand using the classical herring-bone model of the polymer chain with the pendant groups emerging alternately from opposite sides of the backbone. Utilizing concepts from the structure of stereoregular polymers, and more particularly from that of PMMA, it became possible to interpret the X-ray evidence in a satisfactory way by introducing a new conformational model of the polymer chain with the pendant groups all hanging on the same side of the polymer backbone.

With the ribbon-like conformational model used in the present work, it becomes apparent that the liquid crystalline structure involved must be characterized by two directors. The first director, $\mathbf{n}$, is the classical one associated with the optical axis of the pendant groups ; the second one, $\mathbf{p}$, is associated with the polymer backbone. The electro-optical and magnetic properties of the mesomorphic material should depend mainly on the $\mathbf{n}$ director, while the mechanical properties should depend mainly on $\mathbf{p}$. With the ribbon-like conformation, it is also possible to conceive the formation of nematic phases, in which the structural element is the ribbon-like polymer molecule itself. Though not reported to date, this new nematic system should be intrinsically biaxial to take into account the anisotropic shape of the ribbons.

\section{Acknowledgments.}

The authors are grateful to $\mathrm{D}$. Gesbert who registered the X-ray diffraction patterns corresponding to figure 6 .

\section{References}

[1] Blumstein, A., Liquid Crystalline Order in Polymers (Academic Press, New York) 1978.

[2] Ciferri, A., Krigbaum, W. R., Meyer, R. B., Polymer Liquid Crystals (Academic Press, New York) 1982.

[3] Finkelmann, H., Rehage, G., Adv. Polym. Sci. 60/61 (1984) 99.

[4] Shibaev, V. P., Plate, N. A., Adv. Polym. Sci. 60/61 (1984) 173 ;

Lipatov, Yu. S., Tsukruk, V. V., ShIlov, V. V., Rev. Macromol. Chem. Phys. C 24 (1984) 173 ;

TSUKRUK, V. V., SHILOV, V. V., LiPATOV, Yu. S., Konstantinov, I. I., AMERIK, Yu. B., Acta Polym. 33 (1982) 63.

[5] Blumstein, A., Polymeric Liquid Crystals (Plenum Press, New York) 1985.

[6] Zugenmaier, P., Mügge, J., Recent Advances in Liquid Crystalline Polymers, Ed. L. Lawrence Chapoy, Elsevier Appl. Sci. (London) 1985.

[7] Frosini, V., Levita, G., L'upinacci, D., MagagNINI, P. L., Mol. Cryst. Liq. Cryst. 66 (1981) 21.

[8] Davidson, P., Keller, P., Levelut, A. M., J. Phys. France 46 (1985) 939.

[9] Keller, P., Carvalho, B., Cotton, J. P., LamBert, M., Moussa, F., PePy, G., J. Phys. Lett. France 46 (1985) L-1065.

[10] Duran, R., Gramain, Ph., Makromol. Chem. 188 (1987) 2001.

[11] Duran, R., Strazielle, C., Macromolecules 20 (1987) 2853.

[12] Duran, R., Guillon, D., Gramain, Ph., Skoulios, A., J. Phys. France 49 (1988) 121.

[13] Duran, R., Gramain, Ph., Guillon, D., Skoulios, A., Mol. Cryst. Liq. Cryst. Lett. 3 (1986) 23.

[14] Duran, R., Guillon, D., Gramain, Ph.,
Skoulios, A., Makromol. Chem., Rapid Commun. 8 (1987) 181.

[15] Duran, R., Guillon, D., Gramain, Ph., Skoulios, A., Makromol. Chem., Rapid Commun. 8 (1987) 321 ; J. Phys. France 48 (1987) 2043.

[16] Natta, G., Danusso, F., Stereoregular Polymers and Stereospecific Polymerizations (Pergamon Press, Oxford) 1967.

[17] Brochard, F., C.R. Hebd. Séan. Acad. Sci. B 290 (1980) 485.

[18] Dubault, A., Cassagrande, C., Veyssie, M., Mol. Cryst. Liq. Cryst. Lett. 72 (1982) 189.

[19] Skoulios, A., Ann. Phys. Fr. 3 (1978) 421.

[20] Guillon, D., Skoulios, A., J. Phys. France 45 (1984) 607.

[21] Flory, P. J., Proc. R. Soc. London A 234 (1956) 73.

[22] Onsager, L., Ann. N.Y. Acad. Sci. 51 (1949) 627.

[23] Dave, J. S., Vora, R.A., in Liquid Crystals and Plastic Crystals, Eds. G. W. Gray, P. A. Winsor (John Wiley and Sons, N.Y.) 1 (1974) 153.

[24] Alimoglu, A. K., Ledwith, A., Gemmell, P. A., Gray, G. W., LaCY, D., Polymer 25 (1984) 1342.

[25] Bovey, F. A., High Resolution NMR of Macromolecules (Academic Press, New York) 1972.

[26] Odian, G., Principles of Polymerization (John Wiley and Sons, New York) 1981.

[27] WitTMANN, J. C., private communication.

[28] Stroupe, J. D., Hughes, R. E., J. Am. Chem. Soc. 80 (1958) 2341.

[29] D'Alagni, M., De Santis, P., Liquori, A. M., Savino, M., J. Polym. Sci. B 2 (1964) 925.

[30] Liquori, A. M., Anzuno, Q., CoIro, V. M., D'Alagni, M., De Santis, P., Savino, M., Nature (London) 206 (1965) 358. 
[31] Coiro, V. M., de Santis, P., Liquori, A. M., Mazzarella, L., J. Polym. Sci. C 16 (1969) 4591.

[32] Tadokoro, H., Chatani, Y., Kusanagi, H., YoKoYAMA, M., Macromolecules 3 (1970) 441.

[33] Kusanagi, H., Tadokoro, H., Chatani, Y., Macromolecules 9 (1976) 531.

[34] Kusuyama, H., Miyamoto, N., Chatani, Y., TADOKoro, H., Polym. Commun. 24 (1983) 119.

[35] Sundararajan, P. R., Flory, P. J., J. Am. Chem. Soc. 96 (1974) 5025.

[36] Bovey, F. A., Tiers, G. V. D., J. Polym. Sci. 44 (1960) 173.

[37] Higgins, J. S., Allen, G., Brier, P. N., Polymer 13 (1972) 157.

[38] Lowell, R., Windle, A. H., Polymer 22 (1981) 175.
[39] Doucet, J., Levelut, A. M., LAmbert, M., Liebert, L., Strzelecki, J., J. Phys. Colloq. France 36 (1975) C1-13.

[40] Hsieh, H. W. S., Post, B., Morawetz, H., J. Polym. Sci., Polym. Phys. Ed. 14 (1976) 1241.

[41] Plate, N. A., Shibaev, V. P., J. Polym. Sci., Macromol. Rev. 8 (1974) 117.

[42] De Gennes, P. G., The Physics of Liquid Crystals (Clarendon Press, Oxford) 1974.

[43] Mathis, A., Schmitt, A., Skoulios, A., Varoqui, R., Eur. Polym. J. 15 (1979) 255.

[44] Meyer, R. B., Mol. Cryst. Liq. Cryst. 40 (1977) 33.

[45] Birgeneau, R. J., Litster, J. D., J. Phys. Lett. France 39 (1978) L-339.

[46] Benattar, J. J., Moussa, F., Lambert, M., J. Phys. Lett. France 42 (1981) L-67. 